\title{
REA_04 - Clinical validation of a rapid serological test for HIV infection in children 9-24 months old
}

Collaborative Group for Clinical Validation of Diagnostic tests ${ }^{1}$; Luiz Antonio Bastos Camacho ${ }^{2 *}$.

${ }^{1}$ Grupo colaborativo multi-institucional;

${ }^{2}$ Fiocruz/ENSP.

Introduction: The accuracy of serological tests for HIV infection in children less than 2 years old is hindered by maternal antibodies that circulates in the infant's blood for varying time periods.

Objective: To assess the sensitivity and specificity of rapid tests - TR DPP ${ }^{\circ}$ HIV $1 / 2$ and Rapid Immunoblot DPP ${ }$ HIV $1 / 2$, Bio-Manguinhos/Fiocruz - in blood, serum, plasma and oral fluid specimens in children 9-24 months old, to revise current recommendations of rapid tests by the Brazilian Ministry of Health.

Methodology: This was a cross-sectional study in convenient samples of children born from HIV-infected women, recruited in HIV treatment settings in Brazil and in Tanzania. Children were selected according to their infection status confirmed by molecular tests (NAT) as the "gold standard". They provided specimens for rapid tests, and demographic and clinical data. The sensitivity, specificity and likelihood ratios were estimated in age subgroups (9-15, 16-21 and 22-24 months).

Results: From 2017 to 2020, 306 children were enrolled in several centers added to the study in search of eligible children. Only $41 \mathrm{HIV}$-infected children could be recruited, whereas 265 non infected children were available. All but 9 children had been on anti-retroviral treatment (ART). TR-DPP showed 100\% (95\%CI: 85\%-100\%) sensitivity and 92\% (95\%CI: 85\%-96\%) specificity in blood samples of 9-15-monthold children. Sensitivity decreased and specificity increased with age: 78\% (45\%-94\%) and 99\% (93\%$100 \%$ ), respectively, among 22-24-month-old children. The likelihood ratio of a positive test also increased from 12 to 58 in those age groups. A similar pattern was observed for the other specimens, although in oral fluid the sensitivity was only $81 \%$ (60\%-92\%) and specificity was $100 \%(97 \%-100 \%)$ in $9-15$-month-old children, decreasing slightly in the oldest age-group. Rapid Immunoblot DPP showed $100 \%$ sensitivity in 9-15-month-old children decreasing to $89 \%$ among 22-24-month-old children. Conversely, specificity increased with age (93\% to 97\%), being higher for blood samples than for other specimens. "Positive" results were 14 times and 32 times more likely in 9-15-month and 22-24-month old children, respectively. A pattern of age related trend was apparent in most estimates.

Conclusion: These results converge with those obtained with other commercial rapid tests, which also included children with similarly high proportion undergoing ART. Despite the small sample of HIV-infected children and imprecise sensitivity estimates, the results appear to support the role of the combination RTDPP in the screening and rapid Imunoblot tests for confirmation of HIV infection in 9-15-month-old children. In remote settings with limited laboratory resources those rapid tests may provide the elements for timely clinical decisions while the mother and the child are in the health care unit.

Keywords: HIV infection in children; Rapid tests; Clinical validation 